\title{
Correlation between QT-interval Duration and Treatment with Propranolol in Patients with Liver Cirrhosis
}

\section{Lorena Mărieş* and Ioan Maniţiu}

"Lucian Blaga” University of Sibiu, Bulevardul Victoriei 10, Sibiu 550024, Romania

\begin{abstract}
We studied the effect of chronic treatment with propranolol on QT-interval duration in a group of patients with liver cirrhosis. It is known that they may have a secondary cardiac disease, the cirrhotic cardiomyopathy. As several studies have reported a reduction in QT-interval in these cases, we investigated this, knowing that there is frequently a prolonged QT-interval in cirrhotic cardiomyopathy.
\end{abstract}

Keywords: QT-interval; Propranolol; Liver cirrhosis

\section{Introduction}

Cirrhotic cardiomyopathy term is currently defined as:

a. Increased cardiac output at rest, but decreased ventricular response to stimuli.

b. Systolic and/ or diastolic dysfunction.

c. No evidence of left ventricular dysfunction at rest.

d. Electrophysiological abnormalities as prolonged QT-interval and chronotropic incompetence [1-4].

Not all of them are necessary for diagnosis, for instance, only 30 $60 \%$ of patients have a prolonged QT-interval [5].

QT-interval prolongation is the result of abnormal myocardial repolarization and it is occasionally associated with an increased risk of torsades de pointes, a potentially fatal ventricular tachyarrhythmias. Numerous studies have discovered and rediscovered over time this QT-interval prolongation in cirrhotic patients, some documenting a significant correlation between severity of liver disease (indicated by the Child-Pugh score) and corrected QT-interval [5,6].

Non-selective adrenergic antagonists, like propranolol, reduce splanchnic blood flow through various mechanisms: decreased cardiac output, splanchnic vasoconstriction. Henriksen et al. [7] showed that acute administration of propranolol reduces the QT-interval in human subjects with cirrhosis.

The aim of this study was to see if treatment with propranolol induced changes in QT-interval duration in patients studied.

\section{Material and Method}

The study group consisted of 54 patients with liver cirrhosis admitted to the Liver Transplant Clinic in Homburg, Germany, in February-April 2011. Most of the patients (45) were on the waiting list of the clinic for liver transplant. The tests performed were part of their annual check-up.

Patients were examined in terms of hepatology and cardiology (clinical exam, abdominal ultrasound, echocardiography, pulmonary radiography, EKG, and laboratoryinvestigations). The electrocardiogram was performed using the SmartSoft-EKG programme. We selected patients who were treated with propranolol in the study group. We monitored the QT-interval duration in these patients. The programme corrected the QT interval, and all the patients had a normal heart rate, so we didn't use a Bazett correction.

The study group was constituted mostly of men (34), aged 50-59 years, in whom prevailed the alcoholic cirrhosis in the Child B stage. Other etiologies of the cirrhosis were: hepatitis $\mathrm{C}$ and $\mathrm{B}$, colangitis and drugs.

Most patients in our study had no cardiovascular symptoms or signs at the beginning of the study, there were only three who had systolic or diastolic murmurs (mitral and tricuspid insufficiency).

We drafted a patient monitoring record in each patient and we collected all data in a nominal table and inserted them into a database that allows statistical analysis. We used the "Epi Info" programme for creating and managing the database. We included the results in graphics that suggestively highlight the analyzed phenomenon.

Data collected were statistically centralized and processed by age and gender.

\section{Results}

26 out of the 54 patients studied were receiving long-term treatment with propranolol (over 3 years).

Regarding the length of QT-interval in relation to chronic treatment with propranolol, $20(58.8 \%)$ out of the 34 patients with normal QT were treated continuously with propranolol at least the last three years, while 14 patients $(41.42 \%$ ) did not receive propranolol (Figure 1).

Only 1 out of 8 patients with prolonged QT-interval was treated with propranolol $(12.5 \%)$, while 7 patients $(87.5 \%)$ were not receiving this treatment.

$5(41.7 \%)$ out of 12 patients with shortened QT-interval were treated with propranolol, while 7 patients $(58.3 \%)$ did not receive this treatment.

From another point of view, 14 out of 28 patients not treated with propranolol have normal QT-interval, 7 patients have prolonged QTinterval, while the other 7 have shortened QT-interval. 20 out of 26 patients treated with propranolol have normal QT-interval, one patient has prolonged QT-interval and the other 5 have shortened QT-interval.

*Corresponding author: Lorena Mărieş, 15 Dîrstelor Street, Sibiu, Romania, Tel: +40745387973; E-mail: Iorenamaries@yahoo.com

Received October 30, 2013; Accepted December 07, 2013; Published December 17,2013

Citation: Mărieş L, Manițiu I (2013) Correlation between QT-interval Duration and Treatment with Propranolol in Patients with Liver Cirrhosis. Clin Exp Pharmacol 3: 144. doi: $10.4172 / 2161-1459.1000144$

Copyright: ( 2013 Mărieş L, et al. This is an open-access article distributed under the terms of the Creative Commons Attribution License, which permits unrestricted use, distribution, and reproduction in any medium, provided the original author and source are credited. 


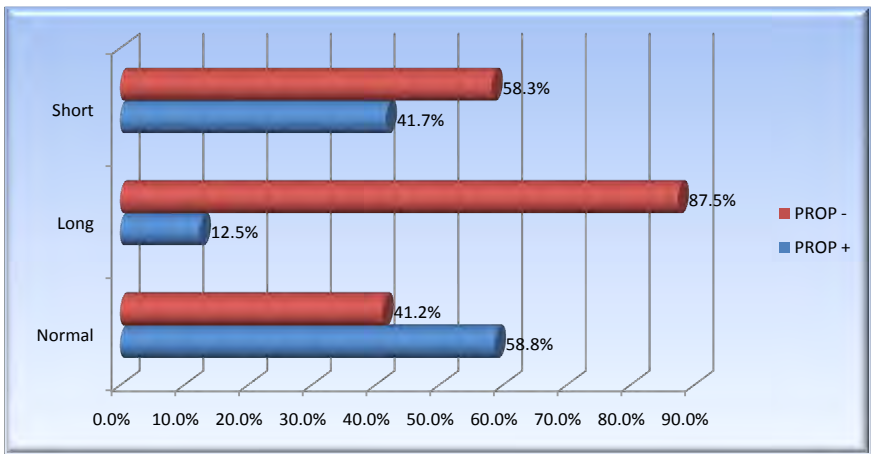

Figure 1: Correlation between QT-interval duration and treatment with propranolol.

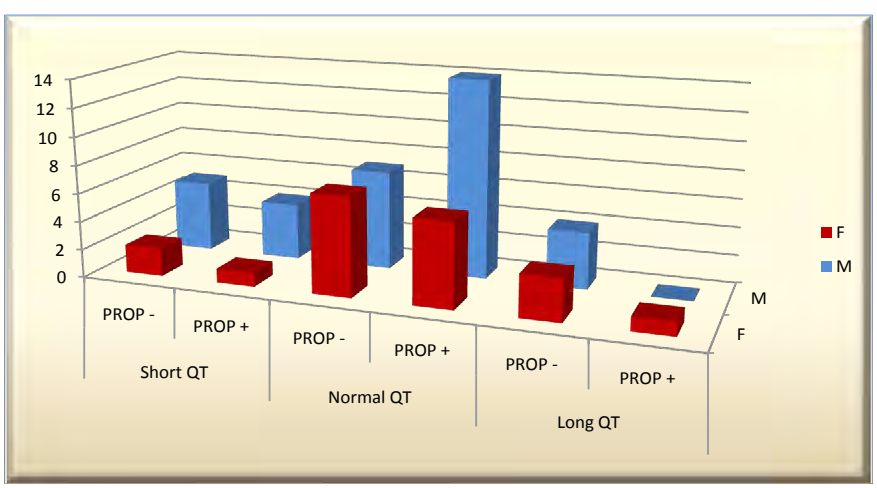

Figure 2: Correlation between QT-interval duration and treatment with propranolol by gender.

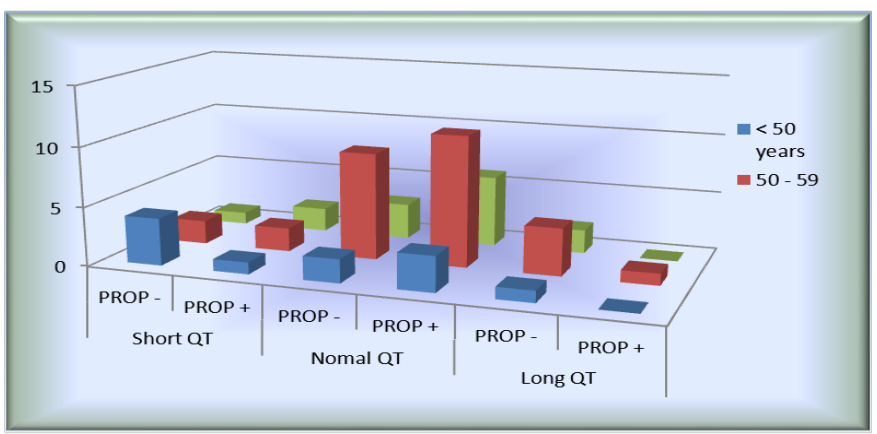

Figure 3: Correlation between QT-interval duration and treatment with propranolol by age group.

Gender analysis of the relationship between QT-interval changes in patients with and without treatment with propranolol, reveals that eight female patients were treated with propranolol and 6 out of these 8 patients have normal QT-interval (75\%), 1 patient has shortened QT-interval (12.5\%) and 1 has prolonged QT-interval (12.5\%). 7 out of 12 women who were not treated with propranolol have normal QTinterval (58.3\% ), 2 have shortened QT-interval (16.7\%), while 3 have prolonged QT-interval (25\%).

18 male patients were treated with propranolol and 14 out of these 18 patients have normal QT-interval (77.8\%), 4 have shortened QTinterval (22.2\%), with no patient with prolonged QT-interval. Only 7 out of 16 male patients not treated with propranolol had normal QT- interval (43.8\%), 5 of them having shortened QT-interval (31.3\%) and 4 with prolonged QT-interval (25\%). It is noted in this case a decrease by half in the number of patients with normal QT-interval among those who were not treated with propranolol, on account of the increase of number of those patients with modified QT-interval, resulting in statistically significant differences (Figure 2).

Regarding the correlation between the QT-interval duration and the chronic treatment with propranolol by age group, 11 out of 14 cases in the age group 50-59 years receiving this treatment have normal QTinterval (78.6\%), only 2 have shortened QT-interval (14.3\%) and one patient has prolonged QT-interval (7.1\%). In the age group above 60 years, 6 out of 8 patients who were treated chronically with propranolol have normal QT-interval (75\%) and 2 have shortened QT-interval (25\%), with no patient with prolonged QT-interval. In the age group below 50 years, 3 out of 4 patients treated with propranolol have normal QT-interval (75\%) and one has shortened QT-interval (25\%), with no patient with prolonged QT-interval.

Among human subjects who were not treated with propranolol, we noticed that in the age group 50-59 years, 9 out of 15 patients have normal QT-interval (60\%), 2 have shortened QT-interval (13.3\%) and 4 have prolonged QT-interval (26.7\%).

In the age group over 60 years, 3 out of 6 patients not treated with propranolol have normal QT-interval (50\%), 1 patient has shortened QT-interval (16.7\%) and 2 patients have prolonged QT-interval (33.3\%). In the age group below 50 years, only 2 out of 7 patients not treated with propranolol have normal QT-interval (28.6\%), 4 having shortened QT-interval (57.1\%) and 1 having prolonged QT-interval (14.3\%) (Figure 3).

It is thus noticed a significantly lower number of cases with normal QT-interval in human subjects not treated with propranolol, unlike those who have received this treatment, in all age groups as follows: a decrease by $25 \%(75-50 \%)$ in the age group above 60 years, a decrease by $18.6 \%(78.6-60 \%)$ in the age group $50-59$ years and a decrease by $46.4 \%(75-28.6 \%)$ in the age group below 50 years.

There is a statistically significant association between the QTinterval duration and the long-term treatment (over 3 years) with propranolol in patients with cardiovascular dysfunction and liver cirrhosis. Thus, $50 \%$ of human subjects who were not treated with propranolol have normal QT-interval, half of them having it short and half of them having it long, while among patients receiving long-term treatment with propranolol, only $23.1 \%$ have modified QT-interval, the majority cases having a shortened QT-interval (19.2\%), so it seems that propranolol protects the normal length of QT-interval primarily by preventing its elongation.

Regarding the gender, the difference between patients treated with propranolol and those who did not receive this treatment is statistically significant in both men and women, with no differences between genders.

Regarding the analysis by age group, differences persist between the two categories of human subjects, without significant differences between age groups.

\section{Conclusions}

QT-interval change occurred more frequently in patients who have not taken Propranolol, which leads us to say that Propranolol protects the normal QT-interval duration primarily by preventing its elongation.

There were a significantly lower number of cases with normal QT- 
Citation: Mărieş L, Manițiu I (2013) Correlation between QT-interval Duration and Treatment with Propranolol in Patients with Liver Cirrhosis. Clin Exp Pharmacol 3: 144. doi:10.4172/2161-1459.1000144

interval in human subjects not treated with Propranolol, unlike those who have received this treatment in all age groups.

Prolonged QT-interval was more frequent in cirrhotic patients who have not taken propranolol.

\section{Acknowledgement}

Research done within POSDRU/6/1.5/S/26 project, co-financed by the European Social Funds by means of the Sectoral Operational Program for the Development of the Human Resources 2007-2013.

\section{References}

1. Liu H, Song D, Lee SS (2002) Cirrhotic cardiomyopathy. Gastroenterol Clin Biol 26: 842-847.

2. Blendis L, Wong F (2000) Is there a cirrhotic cardiomyopathy? Am J Gastroenterol 95: 3026-3028.
3. Møller S, Henriksen JH (2002) Cirrhotic cardiomyopathy: a pathophysiological review of circulatory dysfunction in liver disease. Heart 87: 9-15.

4. Gaskari SA, Honar H, Lee SS (2006) Therapy insight: Cirrhotic cardiomyopathy. Nat Clin Pract Gastroenterol Hepatol 3: 329-337.

5. Baik SK, Fouad TR, Lee SS (2007) Cirrhotic cardiomyopathy. Orphanet J Rare Dis 2: 15.

6. Lee RF, Glenn TK, Lee SS (2007) Cardiac dysfunction in cirrhosis. Best Pract Res Clin Gastroenterol 21: 125-140.

7. Henriksen JH, Bendtsen F, Hansen EF, Møller S (2004) Acute non-selective beta-adrenergic blockade reduces prolonged frequency-adjusted $\mathrm{Q}-\mathrm{T}$ interval (QTC) in patients with cirrhosis. J Hepatol 40: 239-246. 